\title{
Disección coronaria espontánea en el servicio de reanimación. A propósito de un caso
}

\author{
Jonatan Vargas-Caño*, Haizea Montes-Hijosa, Raquel Crespo Sainz-Aja y Karmelo Intxaurraga-Fernández \\ Servicio de Anestesiología, Reanimación y Terapéutica del Dolor, Hospital Galdakao-Usansolo, Galdakao, Vizcaya, España
}

\begin{abstract}
Resumen
La disección espontánea coronaria (DCE) es una causa infrecuente del síndrome coronario agudo (SCA) que afecta en particular a mujeres jóvenes con pocos factores de riesgo ateroescleróticos. Se presenta el caso de una mujer de 47 años que acude al servicio de urgencias por dolor y sensación de peso en la extremidad superior izquierda (ESI). El electrocardiograma inicial muestra un descenso del segmento ST generalizado, por lo que se inicia el tratamiento protocolizado para SCASEST. A las 48 horas se solicitan un cateterismo cardíaco y una tomografía de coherencia óptica (TCO) y se establece el diagnóstico de DCE de la arteria descendente anterior (DA) proximal.
\end{abstract}

Palabras clave: DCE. SCASEST. Antiagregación. Anticoagulación. Hematoma. Intramural.

\section{Spontaneous coronary dissection in the resuscitation service. A case report}

\begin{abstract}
Spontaneous coronary dissection (SCAD) is a rare cause of acute coronary syndrome that essentially affects young women with few atherosclerotic risk factors. We present the case of a 47-year-old woman who comes to the Emergency Department for pain and a sensation of weight in the left upper limb. The initial electrocardiogram shows a generalized decrece in the ST segment, which is why the protocolized treatment for NSTEACS is started. At 48 hours, a cardiac catheterization and an optical coherence tomography is completed, with the diagnosis of SCAD of the proximal anterior descending artery.
\end{abstract}

Key words: SCAD. NSTEACS. Antiplatelet. Anticoagulation. Intramural hematoma.

\section{Introducción}

La disección coronaria espontánea (DCE) se puede definir como el desarrollo agudo de una luz falsa dentro de la pared de la arteria coronaria epicárdica que puede conducir a una limitación del flujo por compresión de la luz coronaria verdadera ${ }^{1}$ y que no se relaciona con aterosclerosis, traumatismo o extensión de una disección aórtica ni es yatrogénica². Por lo tanto, la obstrucción de la arteria coronaria por la formación de un hematoma intramural (HIM) o ruptura de la capa endotelial-íntima produce una lesión miocárdica ${ }^{2}$. Aún se desconoce cuál de los dos mecanismos anteriores de lesión es la noxa inicial, si la ruptura de la capa íntima con el consiguiente HIM y el desarrollo de la
Correspondencia:

*Jonatan Vargas-Caño

E-mail: jona.vargas.c@gmail.com
Disponible en internet: 19-07-2021

Rev Argent Anestesiol. 2020;78:19-23 www.revistaargentinaanestesiologia.com 0370-7792 @ 2021 Federación Argentina de Asociaciones, Anestesia, Analgesia y Reanimación. Publicado por Permanyer México SA de CV. Este es un artículo open access bajo la licencia CC BY-NC-ND (http://creativecommons.org/licenses/by-nc-nd/4.0/). 
falsa luz o si el episodio primario es una hemorragia de los vasos vasculares (vasa vasorum) de la arteria coronaria que origina un HIM que rompe la íntima por elevación de la presión ${ }^{1}$.

\section{Caso clínico}

Se presenta el caso de una mujer de 47 años que llega al servicio de urgencias con sensación de peso y dolor en la ESI irradiado al cuello, junto con náusea, mientras paseaba, y que no cesa con el reposo. El ECG muestra un ritmo sinusal con descenso generalizado de $1.5 \mathrm{~mm}$ del segmento ST. Al ingresar desaparece el dolor por completo y coincide con una mejoría del ECG con rectificación generalizada del ST. De forma inicial se administran $300 \mathrm{mg}$ de AAS y se solicitan cifras de TnT con un primer resultado de $12 \mathrm{ng} / \mathrm{dL}$, que en la seriación aumenta a $131 \mathrm{ng} / \mathrm{dL}$. El resto de la analítica no es relevante.

Como antecedentes personales se identifican abandono de consumo tabáquico reciente (índice acumulado paquetes-año de 15) y trastorno de ansiedad en tratamiento con bromazepam. No tiene antecedentes familiares de cardiopatía isquémica ni muerte súbita.

En el momento de la valoración, la paciente se encuentra sin dolor, hemodinámicamente estable con TAM $>75 \mathrm{mmHg}$ y 70 lpm, y eupneica con saturación arterial adecuada con gafas nasales a $2 \mathrm{Lpm}$. Se realiza ecocardioscopia al pie de cama y se registra una función sistólica global en el límite bajo de la normalidad del ventrículo izquierdo por hipocinesia grave septal medioapical. Por consiguiente, se determina doble antiagregación con carga de ticagrelor $(180 \mathrm{mg})$ y se inicia el tratamiento protocolizado del SCA con captoprilo $(6.25 \mathrm{mg})$, bisoprolol $(1.25 \mathrm{mg})$, atorvastatina (80 mg) y fondaparinux $(2.5 \mathrm{mg})$ antes del ingreso al servicio de reanimación donde se dispone de vigilancia continua.

Durante las primeras horas de ingreso se aumenta de manera paulatina el captoprilo hasta $25 \mathrm{mg}$ cada $8 \mathrm{~h}$ y el bisoprolol de acuerdo con la tolerancia. A lo largo del ingreso, la paciente no vuelve a referir el dolor descrito ni los síntomas vagales, y permanece en todo momento hemodinámicamente estable sin necesidad de fármacos vasoactivos. Al día siguiente del ingreso se detecta un máximo de $533 \mathrm{ng} / \mathrm{dL}$ de TnT y en los días posteriores se conservan unas $\mathrm{TnT}$ elevadas, pero con valores en meseta en torno de $210 \mathrm{ng} / \mathrm{dL}$. A las 48 horas de estancia se efectúa un ecocardiograma reglado y un cateterismo cardíaco. En el ecocardiograma destaca la hipocinesia grave de los segmentos apical y medioapicales septales ya descrita. El cateterismo cardíaco muestra una estenosis del $70 \%$ a nivel proximal de la DA que parece corresponder a un hematoma espontáneo. Para su confirmación se realiza una TCO con el hallazgo de un HIM como causa de la estenosis (Fig. 1 y 2). Una vez establecido el diagnóstico se ajusta el tratamiento antiagregante, se sustituye el ticagrelor por clopidogrel, se suspende el tratamiento anticoagulante planificado y se deciden medidas conservadores de la anomalía. Se solicita analítica con perfil tiroideo que registra valores de la $\mathrm{TSH}, \mathrm{T}_{3}$ y $\mathrm{T}_{4}$ dentro de límites normales. Dada la buena evolución de la paciente durante los tres días tras el ingreso en reanimación, se decide el traslado a planta de hospitalización del servicio de cardiología para seguimiento de evolución.

\section{Discusión}

Se desconoce la verdadera incidencia de la DCE. En realidad, los estudios de Nakashima, et al. ${ }^{3}$ y Nishiguchi, et al. ${ }^{4}$ de 2016 establecen de manera dispar la prevalencia de DCE como origen de SCA al obtener cifras del $0.31 \%$ y $4 \%$, respectivamente. Según el estudio de cohortes multicéntrico de Nakashima, et al. ${ }^{3}$, esta prevalencia es más alta en mujeres jóvenes $\leq 50$ años, como en el caso presentado, en el que la DCE es causa de infarto miocárdico agudo (IAM) hasta en el $35 \%$ de los casos.

Según la revisión de Saw, et al. ${ }^{5}$, los posibles factores de riesgo incluyen displasia fibromuscular (en relación con un porcentaje elevado de anomalías vasculares extracoronarias; en algunos estudios destaca la importancia de su confirmación para el diagnóstico de $\mathrm{DCE}^{6}$ ), período periparto, multiparidad ( $\geq 4$ nacimientos), trastornos del tejido conectivo, afecciones inflamatorias sistémicas y tratamiento hormonal (como anticonceptivos orales y tratamientos para la infecundidad ${ }^{2}$ ). Además, en fecha reciente, en el estudio de Camacho, et al., ${ }^{7}$ se sugirió una posible relación con el hipotiroidismo. Los factores predisponentes para el desarrollo de la DCE descritos son ejercicio intenso o estrés emocional, actividades intensas de tipo Valsalva, consumo de drogas recreativas y tratamiento hormonal radical $^{5}$. En el caso clínico expuesto, solo se recoge el estrés emocional referido por la paciente como posible factor predisponente.

Aunque existe una amplia gama de presentaciones clínicas y gravedad de DCE, los pacientes muestran por lo general una clínica inicial típica de SCA con valores elevados de enzimas cardíacas ${ }^{1}$. Entre el $2 \%$ y el 


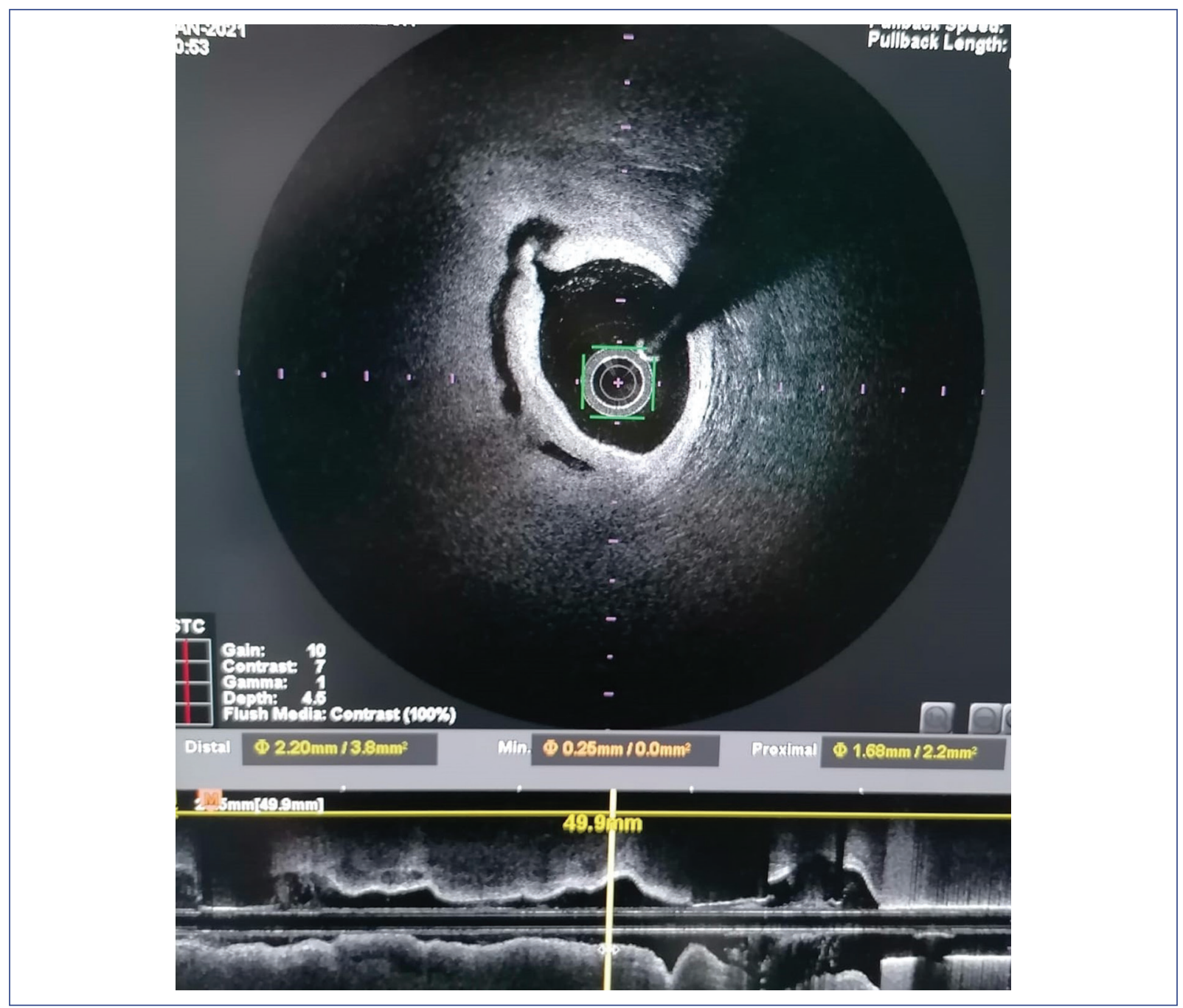

Figura 1. TCO en la que se reconoce una línea de disección como vía de entrada.

$5 \%$ de los pacientes experimenta choque cardiogéni$\mathrm{CO}^{3,8}$. Lo habitual es que se presente en forma de SCASEST con síntomas indicativos de un SCA ateroesclerótico. El signo clínico más frecuente es el dolor torácico (95\%), que no presentaba la paciente, aunque sí refería el resto de los síntomas más comunes: dolor en el brazo (51.5\%), náusea y vómitos $(23.7 \%)$ e irradiación al cuello (22.2\%). Ninguno de los factores anteriores se ha hallado en este caso clínico'.

Los criterios diagnósticos para la definición angiográfica incluyen la presencia de un plano de disección no yatrogénico en ausencia de aterosclerosis coronaria, con cambios típicos de colgajo de la íntima radiolúcido y tinción de contraste. La mayoría de los DCE tiene un estrechamiento largo y difuso en la angiografía debido a un hematoma intramural. En los pacientes en los que el diagnóstico no se confirma con una angiografía, las imágenes intracoronarias con TCO o IVUS (ultrasonidos intravasculares) pueden ser útiles. La RM cardíaca puede ser otra opción útil ${ }^{5}$. La angiografía coronaria por TC en el contexto agudo podría evitar procedimientos invasivos, aunque puede dar lugar a problemas en la detección de lesiones distales ${ }^{1}$. Según lo secunda el estudio de Bastante, et al., ${ }^{9}$ la arteria afectada con más frecuencia es la DA (51\%), tal y como sucede en este caso.

Debido a su baja prevalencia y el reconocimiento reciente de esta anomalía, no existe un consenso para el tratamiento agudo de la DCE. El tratamiento médico adoptado ha sido casi siempre el mismo que el recomendado para el SCA de origen ateroesclerótico. Los objetivos finales son aliviar los síntomas y prevenir la DCE recurrente ${ }^{2}$. Dada la fisiopatología subyacente que afecta a una pared de la arteria coronaria más débil y un episodio hemorrágico primario que conduce 


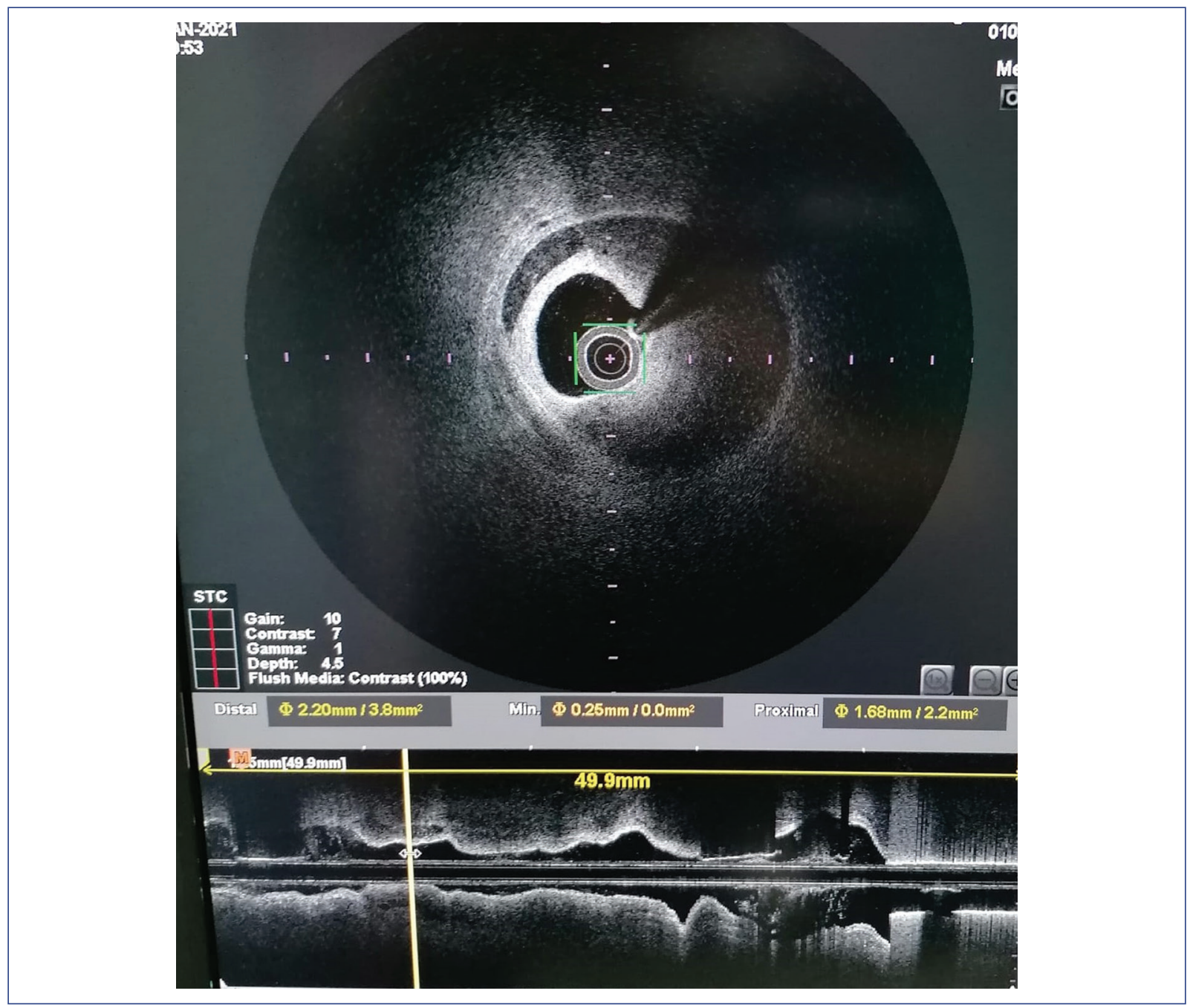

Figura 2. TCO en la que se observa el HIM como causa de la estenosis.

a una HIM parece razonable evitar el tratamiento trombolítico cuando se sospecha DCE. Sin embargo, por falta de sospecha clara al recibir a la paciente, en el caso presentado no se suspendió el fondaparinux antes del cateterismo cardíaco. Por otra parte, los tratamientos antiplaquetarios y su duración son controvertidos. La institución del tratamiento antiplaquetario dual (con AAS y clopidogrel durante la fase aguda) se basa en la posibilidad de que exista un trombo dentro de la luz verdadera ${ }^{1}$, si bien todavía no hay evidencia de su beneficio². Con frecuencia se recomienda AAS de forma indefinida en ausencia de contraindicaciones $^{8}$. En este caso clínico, una vez diagnosticada la DCE, se indicó tratamiento dual de AAS y clopidogrel.

La administración de hipolipemiantes parece inadecuada puesto que no se trata de un origen ateroesclerótico ${ }^{1}$. En realidad, en varios estudios se ha observado un aumento de las recurrencias de DCE en los pacientes tratados con estatinas ${ }^{2}$. Por otro lado, el beneficio de los betabloqueadores se ha extrapolado a partir de la experiencia en la disección aórtica aguda y el SCA aterosclerótico. Están indicados si existe disfunción del ventrículo izquierdo o arritmias y para el tratamiento de la hipertensión, al igual que los IECA y Ios ARA 2. Además, los datos de una cohorte grande muestran un riesgo menor de recurrencias en los sobrevivientes de DCE que toman betabloqueadores ${ }^{10}$. En consecuencia, la paciente permanece en tratamiento con bisoprolol y captoprilo desde el ingreso y se decidió la suspensión de la atorvastatina.

En cuanto a la revascularización, los datos de los estudios observacionales manifiestan un curso clínico benigno de la mayoría de los pacientes con DCE con 
un tratamiento conservador. Además, hay evidencia de un porcentaje elevado de cicatrización completa de los vasos durante el seguimiento ${ }^{1}$. Pueden ser necesarias las intervenciones coronarias percutáneas (ICP) en algunas situaciones, como en la isquemia continua 0 recurrente, la oclusión total del vaso, las arritmias o la inestabilidad hemodinámica. En estos casos, los stents liberadores de fármacos y los andamios bioabsorbibles pueden ser opciones plausibles ${ }^{1}$. La ICP tiene peores resultados en el tratamiento de la DCE, se han descrito tasas mayores de fracaso y se ha relacionado con un aumento del riesgo de disecciones yatrogénicas y de propagación de HIM por la debilidad de las paredes ${ }^{6,8}$. En el caso presentado, dada la situación clínica estable de la paciente, se indicó un tratamiento conservador.

En el trabajo de Lettieri, et al. ${ }^{8}$ se describe una mortalidad de $5.6 \%$ a los seis años después de la DCE. Asimismo, se observan un IAM recurrente (4\%), arritmia ventricular grave $(4 \%)$ y revascularización no planificada (3\%).

\section{Conclusión}

El control agudo de la DCE se ha basado por lo regular en el de un SCA típico. A pesar de ello, y dadas las diferencias fisiopatológicas entre las dos entidades, los tratamiento médico e intervencionista son diferentes, si bien no hay un consenso establecido. En la actualidad se acepta la interrupción de las estatinas y el mantenimiento de los betabloqueadores para una menor tasa de recurrencia. El tratamiento antiagregante (dual o único) continúa en revisión. Por otra parte, el tratamiento de revascularización conservador es por lo regular la conducta de elección si hay estabilidad clínica y ausencia de signos objetivos de isquemia en curso, con resultados favorables.

\section{Financiamiento}

Esta investigación no ha recibido patrocinio específico proveniente de agencias del sector público, sector comercial o entidades sin ánimo de lucro.

\section{Conflicto de intereses}

Los autores declaran no tener ningún conflicto de intereses.

\section{Responsabilidades éticas}

Protección de personas y animales. Los autores declaran que los procedimientos seguidos se conformaron a las normas éticas del comité de experimentación humana responsable y en concordancia con la Asociación Médica Mundial y la Declaración de Helsinki.

Confidencialidad de los datos. Los autores declaran que han seguido los protocolos de su centro de trabajo sobre la publicación de datos de pacientes.

Derecho a la privacidad y consentimiento informado. Los autores han obtenido el consentimiento informado de los pacientes o sujetos referidos en el artículo. Este documento obra en poder del autor de correspondencia.

\section{Bibliografía}

1. García-Guimarães M, Bastante T, Antuña P. Spontaneous coronary artery dissection: mechanisms, diagnosis and management. Eur Cardiol. 2020;15:1-8.

2. Hayes SN, Kim ESH, Saw J. Spontaneous coronary artery dissection: current state of the science: a scientific statement from the American Heart Association. Circulation 2018;137(19):e523-e557.

3. Nakashima T, Noguchi T, Haruta S. Prognostic impact of spontaneous coronary artery dissection in young female patients with acute myocardial infarction: a report from the Angina Pectoris-Myocardial Infarction Multicenter Investigators in Japan. Int J Cardiol. 2016;207:341-348.

4. Nishiguchi T, Tanaka A, Ozaki Y. Prevalence of spontaneous coronary artery dissection in patients with acute coronary syndrome. Eur Heart $\mathrm{J}$ Acute Cardiovasc Care. 2016;5(3):263-270.

5. Saw J. Spontaneous coronary artery dissection. In: Kaski JC, Pellikka P, ed. UpToDate, Waltham, MA. 2021. https://www.uptodate.com/contents/spontaneous-coronary-artery-dissection? source=history widget\#references.

6. García-Guimaraes M, Bastante T, Macaya F. Spontaneous coronary artery dissection in Spain: clinical and angiographic characteristics, management, and in-hospital events. Rev Esp Cardiol (Engl Ed). 2021;74(1):15-23.

7. Camacho-Freire SJ, Díaz JF, Gheorghe LL. Disección coronaria espontánea e hipotiroidismo. Rev Esp Cardiol. 2019;72(8):625-633.

8. Lettieri C, Zavalloni D, Rossini R. Management and long-term prognosis of spontaneous coronary artery dissection. Am J Cardiol. 2015:116(1):66-73.

9. Bastante T, García-Guimaraes M, Muñiz M et al. Manejo contemporáneo de la disección coronaria espontánea. REC: Interventional Cardiology. 2020;2(4):247-255

10. Saw J, Humphries K, Aymong E. Spontaneous coronary artery dissection: clinical outcomes and risk of recurrence. J Am Coll Cardiol. 2017;70(9):1148-1158 